\title{
Surface Chemistry of Aluminium Alloy Slid against Steel Lubricated by Organic Friction Modifier in Hydrocarbon Oil
}

\author{
Ichiro Minami and Ayumi Sugibuchi \\ Department of Chemistry and Bioengineering, Faculty of Engineering, Iwate University, Moriola 020-8551, Japan \\ Correspondence should be addressed to Ichiro Minami, ichiro@iwate-u.ac.jp
}

Received 26 June 2011; Revised 26 September 2011; Accepted 20 October 2011

Academic Editor: Satish V. Kailas

Copyright (๑) 2012 I. Minami and A. Sugibuchi. This is an open access article distributed under the Creative Commons Attribution License, which permits unrestricted use, distribution, and reproduction in any medium, provided the original work is properly cited.

\begin{abstract}
The lubrication mechanism of aluminium alloy slid against steel was investigated from the standpoint of surface chemistry. Low friction and low wear were observed using glycerol mono-olate in a hydrocarbon as lubricant. Increase in the silicon content in the aluminium alloy during rubbing was observed by surface analyses using (1) Auger electron spectroscopy, (2) scanning electron microscopy along with energy dispersive X-ray spectroscopy, and (3) X-ray photoelectron spectroscopy. Mild removal of the passive state (aluminium oxide) from the uppermost surface by the additive during the running-in process was proposed as the lubrication mechanism. The importance of additive chemistry that improves the running-in process was pointed out.
\end{abstract}

\section{Introduction}

Aluminium-based alloys are lightweight and are expected to be energy saving materials for transportation systems. Aluminium is inherently a soft metal; therefore, it is alloyed with silicon and copper in order that it can have sufficient properties. Several additives for the lubrication of aluminium alloys have been reported [1-6]; however, lubricants that improve the tribological properties of aluminium alloys still need optimisation. Conventional additives for steel do not always improve the tribological properties of aluminium alloys under boundary conditions $[7,8]$. These results are attributed to tribo-chemistry related to additives and aluminium surfaces. It should be noted that there are two categories in this chemistry: the first is poor reactivity of the additive with the surface (kinetic issue), and the second is inadequate tribological properties of the boundary film formed on the surface by tribochemical reactions (thermodynamic issue). These problems were studied experimentally by a combination of a well-designed tribotest with surface analysis [8]. Lubricant chemists have to consider these categories while developing an appropriate lubricant for an individual material.

Lubrication of aluminium alloys by alcohols is well known [9]. Formation of an amorphous-like boundary film composed of aluminium alkoxide has been detected by chemical analyses [10]. The direct interaction between alcohol and the rubbed aluminium surface has also been analytically detected [11]. Effects of the molecular structure of lubricant on the tribological properties of aluminium alloys are being debated. It has been reported that straightchain primary alcohols having more than 12 carbons are better lubricants than 1,2-diols [11]. Many researchers have pointed out the importance of bifunctionality in the lubricant molecule. A strong interaction between the material and the lubricant molecule through a cyclic complex has been proposed. This leads to improvement in the tribological properties [12]. Thermal stability of aluminium complexes derived from 1,2-diols and 1,3-diols has been pointed out [13]. Effects of molecular structure in 1,2-diols and 1,3-diols on the tribological properties of aluminium-steel contact have been studied systematically [14]. Friction and wear were reduced by 1,2-diols and their derivatives and, to a lesser extent, by 1,3- and 1,4-diols.

In spite of the aforementioned advantages, alcohols are unsuitable as base fluids and the application of neat alcohols as base fluids is problematic. Auto-oxidation of alcohols, which occurs even during storage, yields carboxylic acids; these acids may corrode metallic materials. In addition, alcohols sometimes damage polymers used as parts in 


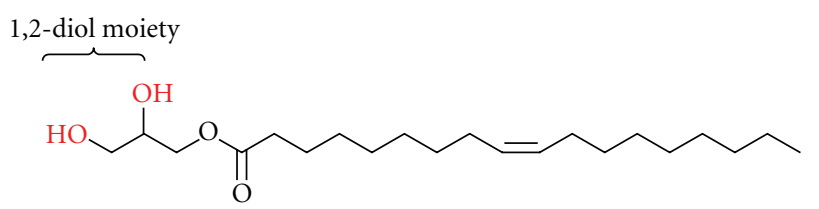

Figure 1: Structure of GMO.

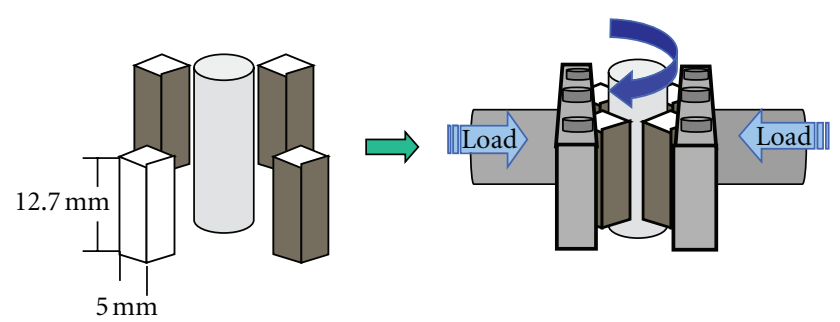

FIgURE 2: Configuration of specimen of tribotester.

TABLE 1: Viscosity of base fluid.

\begin{tabular}{lcc}
\hline \multirow{2}{*}{ Sample } & \multicolumn{2}{c}{ Viscosity, $\mathrm{mm}^{2} \cdot \mathrm{s}^{-1}$} \\
& $40^{\circ} \mathrm{C}$ & $100^{\circ} \mathrm{C}$ \\
\hline Squalane & 18.6 & 4.07 \\
PAO & 250 & 30.0 \\
\hline
\end{tabular}

machinery. The reactivity of alcohols is considered to cause these problems. One practical solution to reduce the reactivity is to dilute alcohols in neutral solvents. Lubricant chemists are familiar with this method as additive technology. Therefore, we attempted to use alcohols as additives for lubricating aluminium alloys. In our preliminary study, we evaluated several alcohols as additives in hydrocarbon fluids. Among them, we found that glycerol mono-olate or 2,3-dihydroxypropyl 9Z-octadecenoate (GMO, Figure 1) provides good lubricity when applied to aluminium alloy slid against steel. The additive has a unique chemical structure: a straight-chain ester having a 1,2-diol moiety at the terminal of the molecule. The 1,2-diol structure is expected to be an anchor on the aluminium surface by forming cyclic complexes [12] comprising an aluminium atom, two oxygen atoms, and two carbon atoms. The straight chain moiety provides a densely packed boundary film that reduces friction. In this study, we investigated the lubrication mechanism of aluminium-silicon alloy by GMO in a hydrocarbon from the standpoint of surface chemistry.

\section{Experimental}

2.1. Chemicals and Materials. Squalane and poly-alphaolefins (PAOs) were employed as base fluids. The former was selected as a pure hydrocarbon in fundamental research. Their viscosities are listed in Table 1. The structure of the additive, GMO, is shown in Figure 1. Samples for use in the tribotest were prepared by dissolving GMO in hydrocarbon oil at a concentration of $50 \mathrm{mmol} \mathrm{kg}-1$ (1.8 mass\%). The tribomaterials employed in this work are for bearing materials.

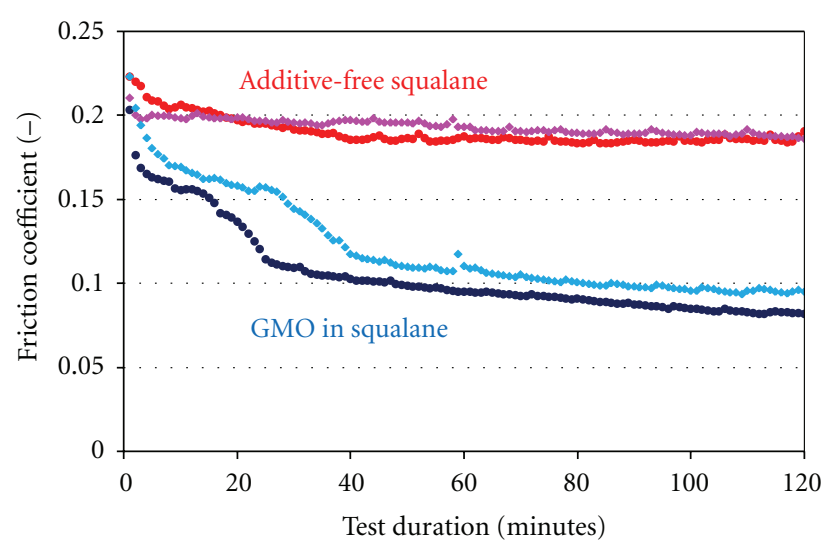

FIgURE 3: Friction trace for squalane.

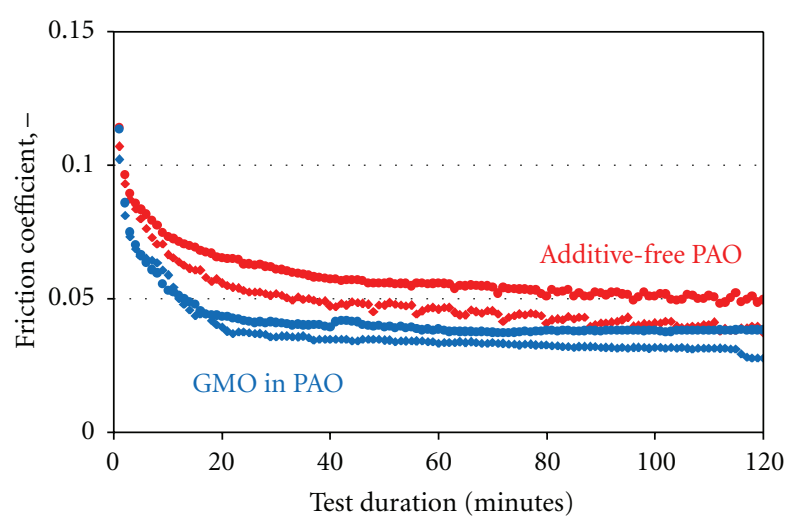

Figure 4: Friction trace for PAO.

Properties and compositions of the materials are listed in Table 2.

2.2. Tribotest. The tribological properties of samples were evaluated by a cylinder and an assembled four-block-type tribotester (Figure 2). This is a unique method designed for evaluating the compatibility of a lubricant with the tribomaterial from the viewpoint of tribochemistry [8]. The test conditions are listed in Table 3 . These conditions were chosen to simulate bearing operation. Low sliding velocity was applied to trace changes in surface chemistry during running-in process. Friction was monitored throughout the test. After the test, the resultant blocks were ultrasonically cleaned in hexane and their surfaces were studied by Auger electron spectroscopy (AES), scanning electron microscopy along with energy dispersive X-ray spectroscopy (SEMEDX), and X-ray photoelectron spectroscopy (XPS). The resultant oil containing wear particle was diluted with hexane, and the liquid phase was removed by decantation. Wear particles were washed with hexane for three times and dried in air. Then the wear particles were collected for SEM-EDX analysis. AES was taken on PHI Instrument Model-680 at electron beam energy of $5 \mathrm{kV}$, test area of $50 \times 50 \mu \mathrm{m}$, and analysis depth of 3-4 $\mathrm{nm}$. SEM-EDX was taken on Elionix model EXM-3500 at accelerating voltage of $25 \mathrm{kV}$, test area of 
TABLe 2: Properties and composition of tribo-material.

\begin{tabular}{lcccccccccccc}
\hline \multirow{2}{*}{ Material } & \multirow{3}{*}{ Hardness } & \multicolumn{2}{c}{ Surface roughness, Ra $\mu \mathrm{m}$} & \multicolumn{1}{c}{ Contents of element, mass\% } \\
& & Block & Cylinder & $\mathrm{Fe}$ & $\mathrm{Cu}$ & $\mathrm{Al}$ & $\mathrm{C}$ & $\mathrm{Si}$ & $\mathrm{Mn}$ & $\mathrm{Cr}$ & $\mathrm{Pb}$ & $\mathrm{Sn}$ \\
\hline $\begin{array}{l}\text { Steel } \\
\begin{array}{l}\text { Aluminium } \\
\text { alloy }\end{array}\end{array}$ & $\mathrm{HRC} 60$ & - & $<0.10$ & Balance & & & 4.6 & 0.47 & $<0.5$ & 1.5 & & \\
\hline
\end{tabular}

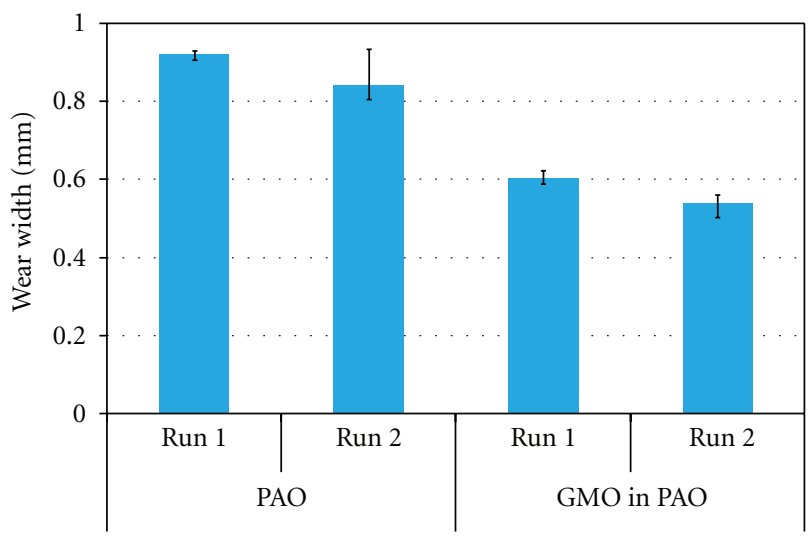

Figure 5: Wear on aluminium block after tribotest for $120 \mathrm{~min}$.

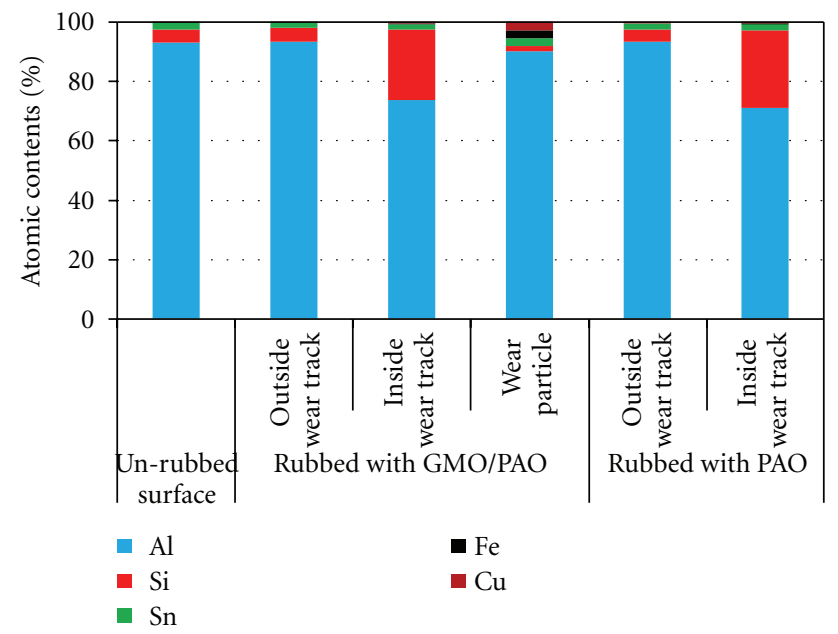

FIGURE 6: Concentration of elements on surface obtained by SEMEDX analysis.

$0.1 \times 0.1 \mathrm{~mm}$, electron beam incidence of 76 degree, emission angle of 29 degree, and analysis depth of $1 \mu \mathrm{m}$. XPS was taken on PHI Instruments Quantum-2000 by X-ray source of monochromated-Al-K- $\alpha$ ray $(1486.6 \mathrm{eV})$ at $20 \mathrm{~W}$, test area of $\phi 100 \mu \mathrm{m}$ ellipse, emission angle of 45 degree, and analysis depth of $4 \mathrm{~nm}$.

\section{Results and Discussion}

A solution of GMO in squalane reduced friction considerably, as shown in Figure 3. GMO fairly improved the tribological properties of the practical base oil, PAO (Figure 4); the coefficient of friction was reduced and

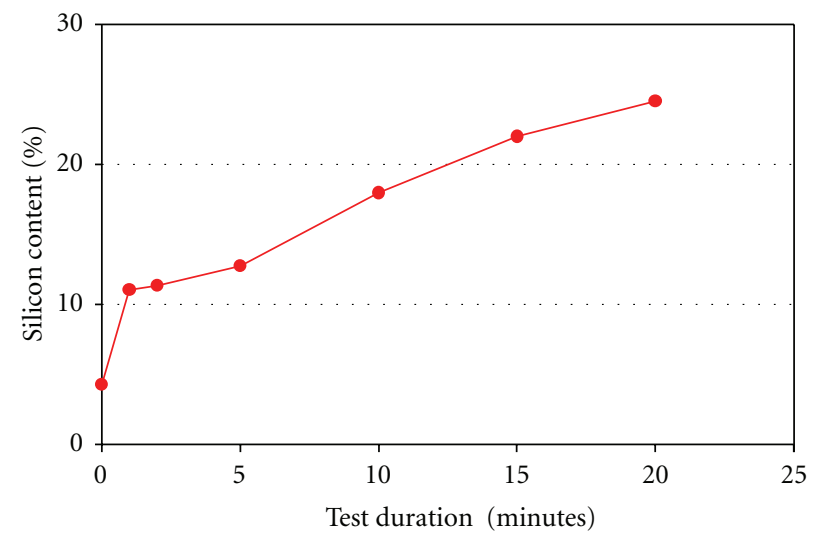

Figure 7: Increase in silicon concentration on aluminium block during running-in period (GMO in $\mathrm{PAO})$.

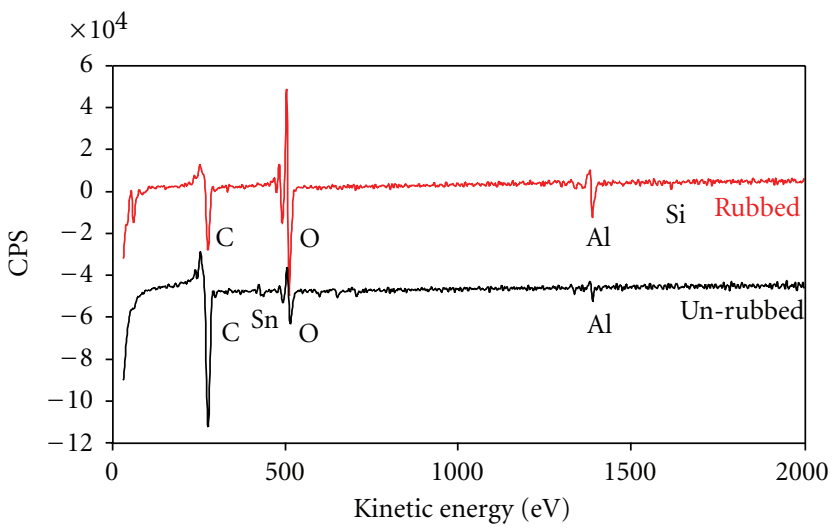

FIGURE 8: AES spectrum of rubbed surface (GMO in PAO for $120 \mathrm{~min})$.

fluctuation of friction was prevented after a running-in period (approximately $20 \mathrm{~min}$ ). The additive also reduced wear of the aluminium block in the tribotest performed for 120 min (Figure 5). The resultant blocks were analyzed by SEM-EDX to study the elemental content on the surface. A considerable increase in silicon content and decrease in the aluminium content were observed on the wear track [15], whereas no content change was observed outside the wear track (Figure 6). Wear particles generated during the tribotest were collected and their elemental composition was also analyzed by SEM-EDX. In addition to aluminium as the major component, detectable amounts of copper and tin were found therein. Iron that had been worn off from the steel cylinder was also found. However, the concentration 

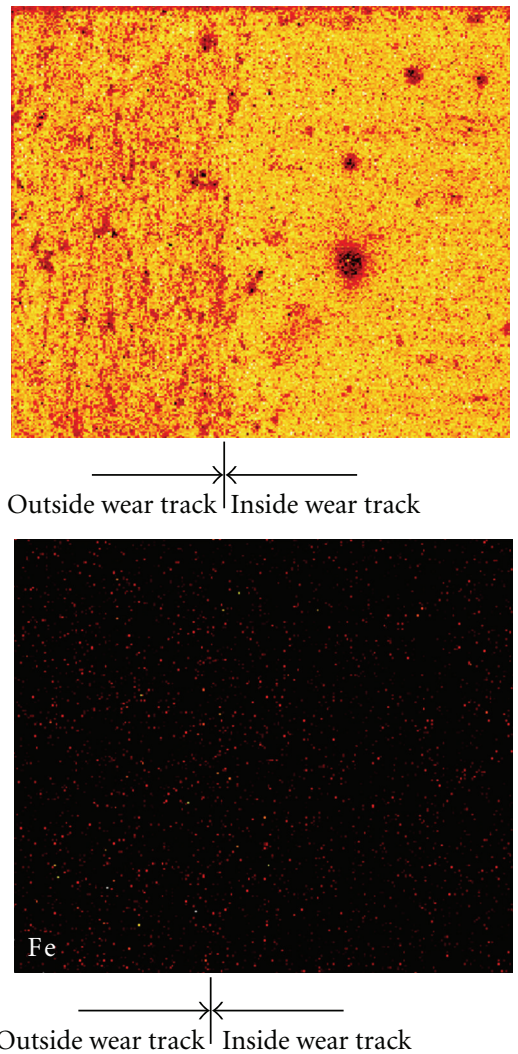
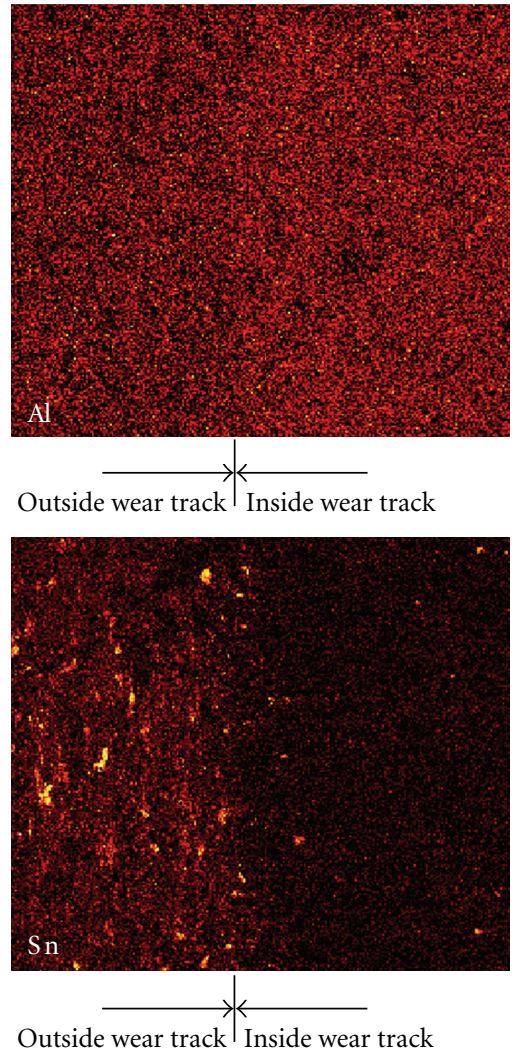
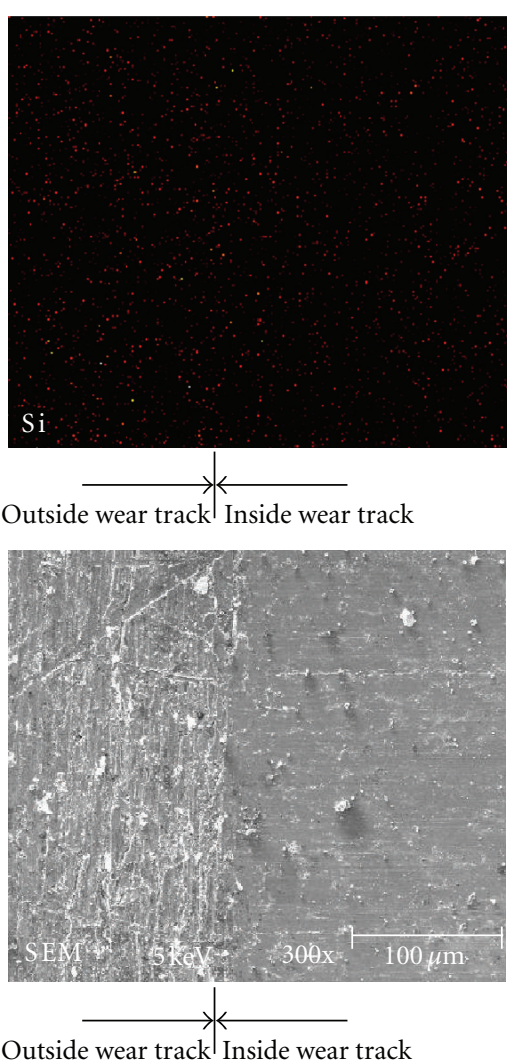

Figure 9: Chemical mapping by AEX (GMO in PAO for $120 \mathrm{~min}$ ).

TABLe 3: Conditions for tribotest.

\begin{tabular}{|c|c|c|}
\hline \multirow{8}{*}{$\begin{array}{l}\text { Operation } \\
\text { parameters }\end{array}$} & Applied load, $\mathrm{N}$ & $1.0 \times 10^{2}$ \\
\hline & Maximum contaxt stress, GPa & $1.8 \times 10^{-1}$ \\
\hline & Hertzian contact width, mm & $2.0 \times 10^{-2}$ \\
\hline & Rotation of cylinder, rpm & 145 \\
\hline & Sliding velocity, $\mathrm{m} \mathrm{s}^{-1}$ & $4.8 \times 10^{-2}$ \\
\hline & Oil temperature, ${ }^{\circ} \mathrm{C}$ & $20-25$ \\
\hline & Oil supply, mg s ${ }^{-1}$ & 0.5 \\
\hline & Test duration, min & 120 \\
\hline \multirow{2}{*}{ Test cylinder } & Material & Steel \\
\hline & Size, $\mathrm{mm}$ & $6.35 \Phi \times 32 \mathrm{~L}$ \\
\hline \multirow{2}{*}{ Test block } & Material & Aluminum alloy \\
\hline & Size, $\mathrm{mm}$ & $5.0 \times 5.0 \times 12.7$ \\
\hline
\end{tabular}

of silicon in the wear particles was less than that in the original material. The results indicate that silicon in the tribo-material remained on the surfaces, whereas the other components $(\mathrm{Al}, \mathrm{Cu}, \mathrm{Sn})$ were worn off by rubbing. It seems that soft metals (such as $\mathrm{Al}$ ) are easy to wear off in comparison with hard metals (such as $\mathrm{Si}$ ). The chemical compositions on the surfaces rubbed with GMO-PAO and additive-free PAO are quite similar. This indicated that the additive had no hand in changing the elemental content on the surface. However, additive-free PAO yielded rough worn surfaces that were unsuitable for further analyses by AES and
XPS. The function of the additive was to provide a smooth worn surface (see SEM micrograph in Figure 9) as well as to reduce the amount of wear. From observations of the tribotest, it was assumed that GMO makes the wear milder, resulting in a smooth worn surface.

We further investigated the increase in silicon content during the running-in process of the tribotest. The silicon content on the worn surface was traced during the initial 20 min of the test. As shown in Figure 7, it increased rapidly during the first minute of the test and then linearly increased during the next $20 \mathrm{~min}$. Since the concentration of silicon on rubbed surfaces after $120 \mathrm{~min}$ of the tribotest fell in the range of $23-25 \%$ (Figure 6), we concluded that the surface chemistry had reached the steady state within 20 min of rubbing under these conditions. The friction trace (Figure 4) also indicated the steady state of low friction after 20 min of rubbing. The results support the importance of the chemical content on a surface in reducing friction.

SEM-EDX provides average chemical contents in subsurfaces of $1 \mu \mathrm{m}$ depth. In contrast, AES and XPS are surface-sensitive analyses that provide the surface chemistry of nanometre depth (uppermost surfaces). Differences in relative concentrations of elements on surfaces were observed by these instrumental analyses (Table 4 ). In contrast to the results obtained by SEM-EDX, silicon content on the nonrubbed surface was lower than the detection limit of AES and XPS analyses (see also Figures 8,9 and 11). These results indicate that the uppermost surfaces of nonrubbed 
TABLE 4: Contents of element on surface.

\begin{tabular}{|c|c|c|c|c|c|c|c|c|c|}
\hline \multicolumn{2}{|c|}{ Analysis } & \multicolumn{8}{|c|}{ Relative contents, atomic\% } \\
\hline \multirow{2}{*}{ Method } & \multirow{2}{*}{ Depth } & \multicolumn{4}{|c|}{ Rubbed surface } & \multicolumn{4}{|c|}{ Non-rubbed surface } \\
\hline & & $\mathrm{Al}$ & $\mathrm{Si}$ & Sn & $\mathrm{Fe}$ & $\mathrm{Al}$ & $\mathrm{Si}$ & Sn & $\mathrm{Fe}$ \\
\hline AES & $3-4 \mathrm{~nm}$ & 75 & 25 & $\mathrm{n} / \mathrm{d}$ & $\mathrm{n} / \mathrm{d}$ & 100 & $\mathrm{n} / \mathrm{d}$ & $\mathrm{n} / \mathrm{d}$ & $\mathrm{n} / \mathrm{d}$ \\
\hline XPS & $4 \mathrm{~nm}$ & 82 & 14 & 2 & 2 & 91 & $\mathrm{n} / \mathrm{d}$ & 9 & $\mathrm{n} / \mathrm{d}$ \\
\hline SEM-EDX & $1 \mu \mathrm{m}$ & 74 & 24 & 2 & trace & 93 & 4 & 3 & $\mathrm{n} / \mathrm{d}$ \\
\hline
\end{tabular}

$\mathrm{n} / \mathrm{d}$ : not detected.

trace: less than $1 \%$.

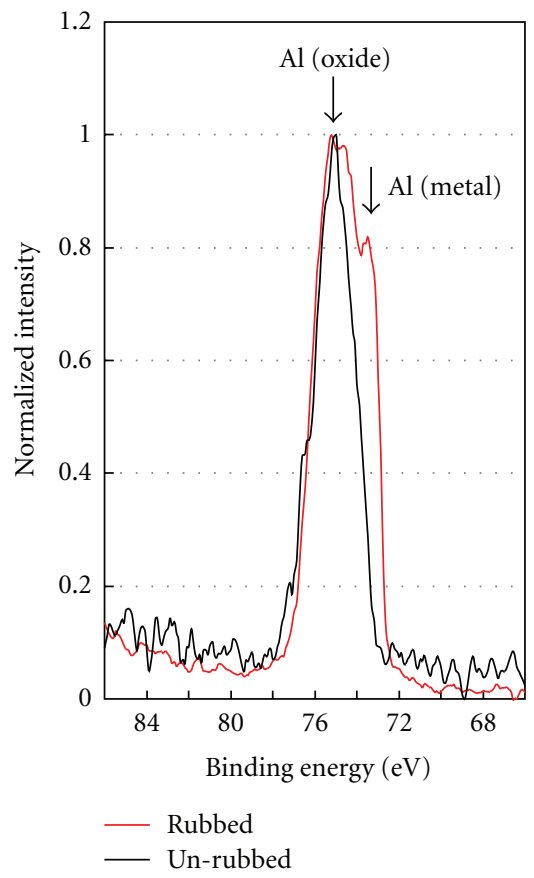

Figure 10: XPS spectrum of rubbed surface: Al 2p (GMO in PAO for $120 \mathrm{~min}$ ).

surfaces were covered with aluminium and a small amount of silicon, if any. AES and XPS analyses showed that the rubbing process increased the silicon content also on the uppermost surfaces. The silicon concentration determined by AES was higher than that determined by XPS. It should be noted that the accuracy of quantification by AES is inferior to that by XPS [16]. Another advantage of XPS is that it yields the chemical state of the substances on the uppermost surface. The spectra shown in Figures 10, 11, 12 and 13 indicate that the nonrubbed area was covered with aluminium oxide and tin oxide, while considerable amounts of aluminium, silicon, and tin in the elemental (metallic) state existed on the rubbed surface.

The tribological process changed the chemical contents on the surface. First, the concentration of silicon on the subsurface $(1 \mu \mathrm{m}$ in depth) increased by 4 to 5 times. Second, considerable amounts of aluminium, tin, and silicon existed in the metallic state (not as oxides) on the rubbed uppermost surface, whereas only oxides of aluminium and tin were found on the nonrubbed surface. These results can

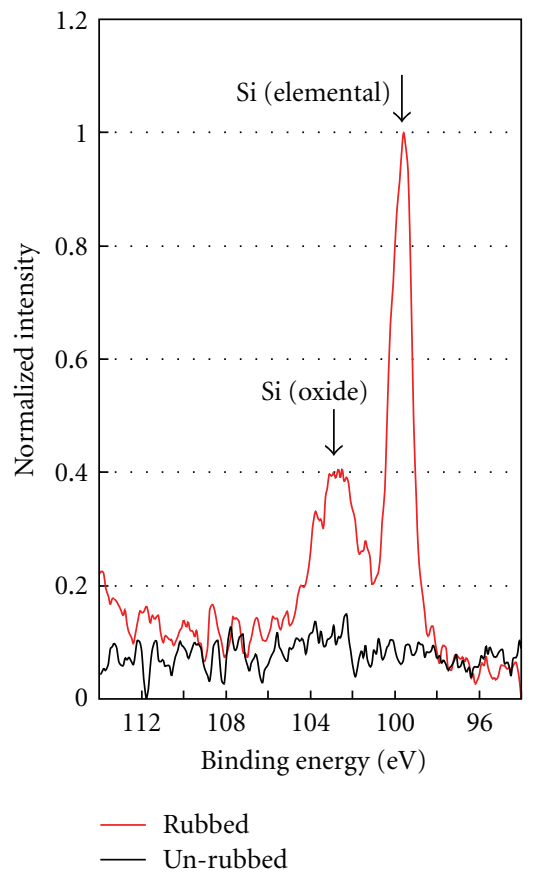

Figure 11: XPS spectrum of rubbed surface: Si 2p (GMO in PAO for $120 \mathrm{~min}$ ).

be explained by the removal of metal oxides existing on the uppermost surfaces through rubbing. As a consequence, metals were exposed on the uppermost surfaces. Mohs hardness of metals and minerals is 2.9 for $\mathrm{Al}, 9.0$ for $\mathrm{Al}_{2} \mathrm{O}_{3}$ (alndum), 7.0 for $\mathrm{Si}, 7.0$ for $\mathrm{SiO}_{2}$ (quartz), 1.8 for $\mathrm{Sn}$, and 6.5 for $\mathrm{SnO}_{2}$ (cassiterite) [17]. Since metallic aluminium and tin are softer than their corresponding oxides, the existence of these metals on the uppermost surface is beneficial for reducing friction. Increase in silicon content on the uppermost surface makes the material harder; this is beneficial for preventing wear [15]. A detectable amount of iron, which was transferred from the counter steel surface, was found on the rubbed surface by XPS.

The third remark is about the chemical content of the wear particles obtained by SEM-EDX. In addition to aluminium and iron as major components of the tribomaterials, considerable amounts of tin and copper were found. In contrast, the concentration of silicon in wear particles was much lower than that in the original material. These results suggest certain selectivity of elements in the 


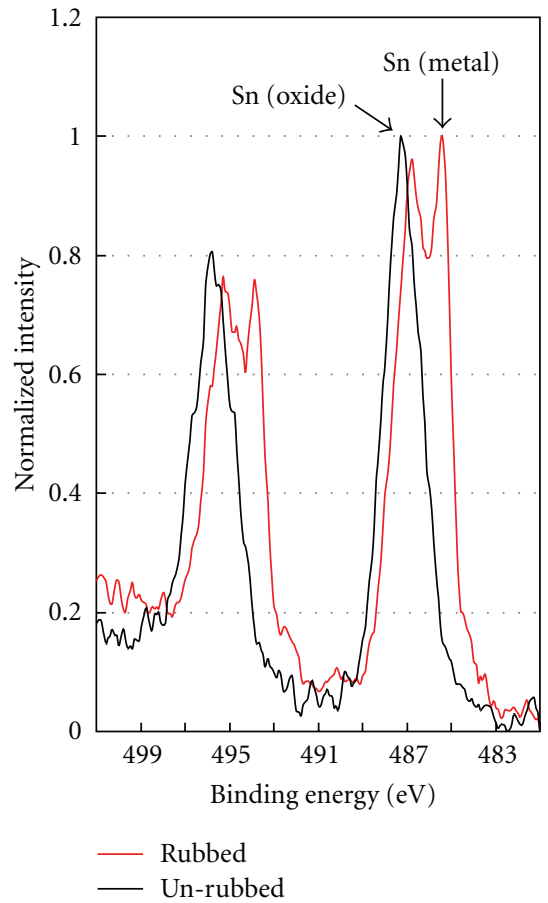

Figure 12: XPS spectrum of rubbed surface: Sn 3d (GMO in PAO for $120 \mathrm{~min}$ ).

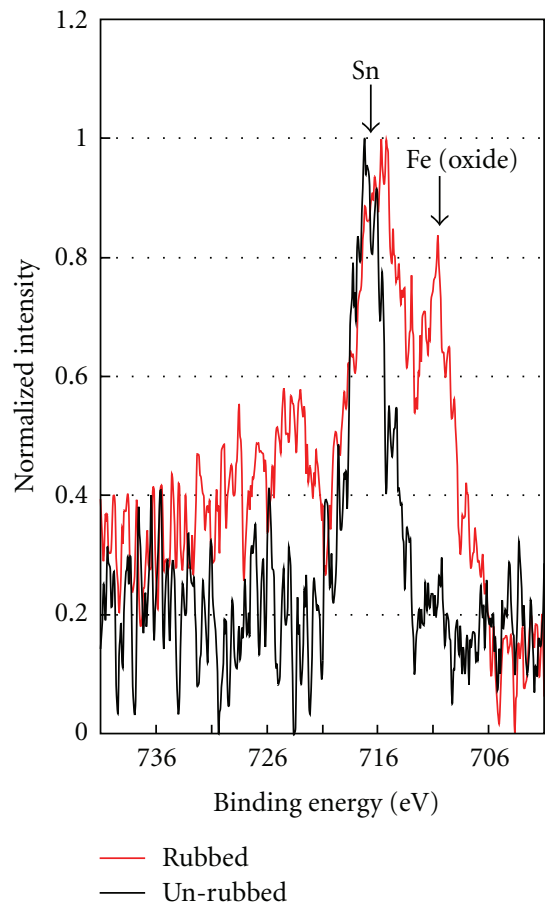

FIGURE 13: XPS spectrum of rubbed surface: Fe 2p (GMO in PAO for $120 \mathrm{~min}$ ).

wear process under these conditions. These results are summarized in Figure 14.

It has been reported that 1,2-diols and 1,3-diols as additive in mineral oil improve lubrication of Al-Si alloy slid against steel by forming a molecular film on the surface [13]. This mechanism is not probable in the present case;

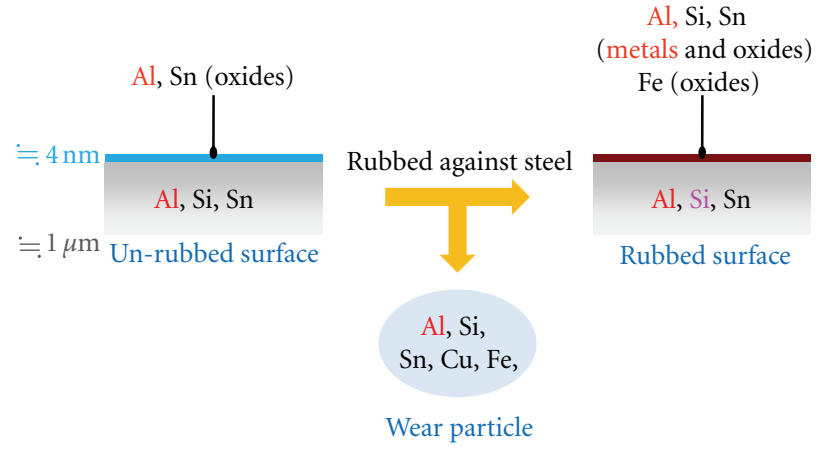

FIgURE 14: Proposed mechanisms based on surface chemistry.

the thickness of the boundary film is approximately $2 \mathrm{~nm}$ if an oriented, densely packed adsorption film of GMO is applied uniformly. This should result in an increase in carbon content on the uppermost surfaces. However, the carbon content on the rubbed surface decreased significantly by rubbing, as shown in Figure 8.

Finally, it should be emphasized that an appropriate combination of analytical techniques leads to better understanding of tribochemistry under boundary lubrication conditions. In this study, the spatial resolution and chemical resolution of surface analyses were of importance.

\section{Conclusions}

The tribological properties of aluminium alloy slid against steel were investigated from the standpoint of surface chemistry.

(1) Glycerol mono-olate reduced friction and wear when it was added to hydrocarbon oil.

(2) Changes in the chemical content on the surface occurred during the tribotest. A significant increase in the concentration of silicon on the rubbed surface was observed by SEM-EDX. This contributes to improving wear-preventing properties. Considerable amounts of metallic aluminium, silicon, and tin on the uppermost surface were found by XPS.

(3) Since these metals are softer than their oxides, they are beneficial for friction reduction.

(4) The additive, GMO, was considered to mildly remove the passive state of aluminium oxide from the surface during the running-in process.

(5) Each surface analysis has individual spacial and chemical resolutions. The combination of the results by these analyses indicates a proposed mechanism (Figure 14). A systematic approach by multisurface analyzing is of importance in studying tribochemistry.

\section{Nomenclature}

AES: $\quad$ Auger electron spectroscopy

GMO: $\quad$ Glycerol mono-olate, see also Figure 1

PAO: $\quad$ Poly-alpha-olefins, see also Table 1

SEM-EDX: Scanning electron microscope attached with energy dispersive X-ray spectroscopy

XPS: X-ray photoelectron spectroscopy. 


\section{Acknowledgments}

This study was supported by a Grant-in-Aid for Scientific Research (22656040) from the Ministry of Education, Culture, Sports, Science and Technology, Japan. The authors are grateful to Taiho Kogyo Co., Ltd., for providing the aluminium alloy, as well as Idemitsu Kosan K.K. for providing the lubricants.

\section{References}

[1] W. Yong and X. Qunji, "Friction and wear characteristics of p-containing antiwear and extreme pressure additives in the sliding of steel against aluminum alloy," Wear, vol. 188, no. 12, pp. 27-32, 1995.

[2] Y. Wan and Q. Xue, "Effect of phosphorus-containing additives on the wear of aluminum in the lubricated aluminumon-steel contact," Tribology Letters, vol. 2, no. 1, pp. 37-45, 1996.

[3] Y. Hu and W. Liu, "Tribological properties of alcohols as lubricating additives for aluminum-on-steel contact," Wear, vol. 218, no. 2, pp. 244-249, 1998.

[4] C. Kajdas and W. Liu, "Tribochemistry of aluminium and aluminium alloy systems lubricated with liquids containing alcohol or amine additive types and some other lubricants-a review," Lubrication Science, vol. 16, no. 3, pp. 267-292, 2004.

[5] M. A. Nicholls, P. R. Norton, G. M. Bancroft, and M. Kasrai, "X-ray absorption spectroscopy of tribofilms produced from zinc dialkyl dithiophosphates on Al-Si alloys," Wear, vol. 257, no. 3-4, pp. 311-328, 2004.

[6] A. Neville, A. Morina, T. Haque, and M. Voong, "Compatibility between tribological surfaces and lubricant additives-how friction and wear reduction can be controlled by surface/lube synergies," Tribology International, vol. 40, no. 10-12, pp. 1680-1695, 2007.

[7] M. A. Nicholls, P. R. Norton, G. M. Bancroft, M. Kasrai, G. De Stasio, and L. M. Wiese, "Spatially resolved nanoscale chemical and mechanical characterization of ZDDP antiwear films on aluminum-silicon alloys under cylinder/bore wear conditions," Tribology Letters, vol. 18, no. 3, pp. 261-278, 2005.

[8] I. Minami, A. Yamazaki, H. Nanao, and S. Mori, "A cylinder and assembled four-block type tribo-test: novel method to study tribo-chemistry of lubricant and material," Tribology Online, vol. 2, no. 1, pp. 40-43, 2007.

[9] R. S. Montgomery, "The effect of alcohols and ethers on the wear behavior of aluminum," Wear, vol. 8, no. 6, pp. 466-473, 1965.

[10] S. Hironaka and T. Sakurai, "The effect of pentaerythritol partial ester on the wear of aluminum," Wear, vol. 50, no. 1, pp. 105-114, 1978.

[11] F. Wiltord, J.-M. Martin, T. Le Mogne, F. Jarnias, M. Querry, and P. Vergne, "Reaction mechanisms of alcohols on aluminum surfaces," Tribologia, vol. 37, no. 6, pp. 7-20, 2006.

[12] B. W. Hotten, "Bidentate organic oxygen compounds as boundary lubricants for aluminum," Lubrication Engineering, vol. 30, no. 8, pp. 398-402, 1974.

[13] Y. Wan, W. Liu, and Q. Xue, "Effects of diol compounds on the friction and wear of aluminum alloy in a lubricated aluminum-on-steel contact," Wear, vol. 193, no. 1, pp. 99-104, 1996.

[14] S. Igari, S. Mori, and Y. Takikawa, "Effects of molecular structure of aliphatic diols and polyalkylene glycol as lubricants on the wear of aluminum," Wear, vol. 244, no. 1-2, pp. 180-184, 2000.

[15] F. A. Davis and T. S. Eyre, "The effect of silicon content and morphology on the wear of aluminium-silicon alloys under dry and lubricated sliding conditions," Tribology International, vol. 27, no. 3, pp. 171-181, 1994.

[16] J. F. Watts and J. Wolstenholme, An Introduction to Surface Analysis by XPS and AES, John Wiley and Sons, 2003.

[17] J. R. Rydberg, "Hardness of elementary substances," Zeitschrift fuer Physikalische Chemie Stoechiometrie und Verwandtschaftslehre, vol. 33, pp. 353-359, 1900. 

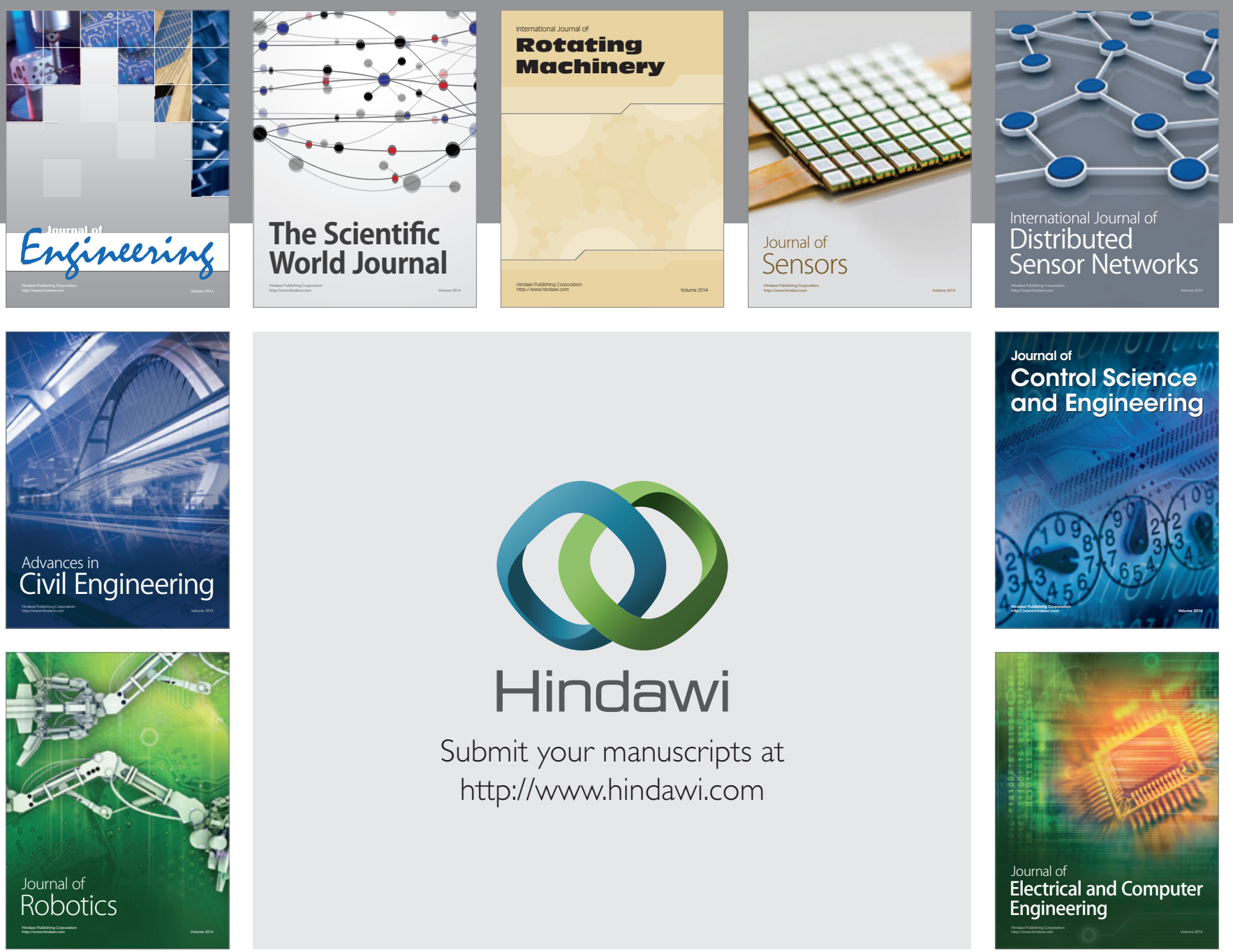

Submit your manuscripts at

http://www.hindawi.com
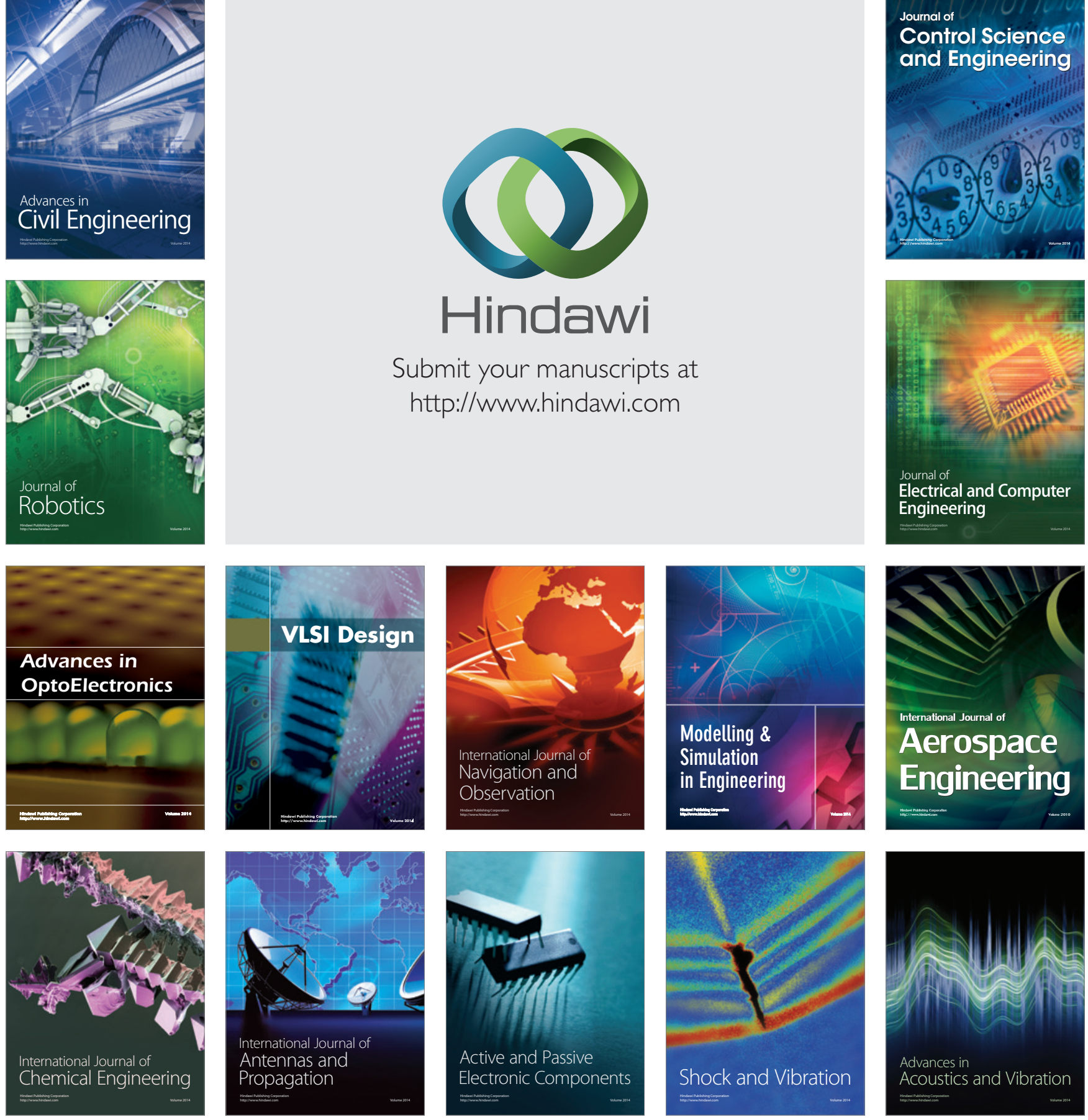